HUB-EP-95/34

hep-th/9512165

\title{
Extended supersymmetry with gauged central charge
}

\author{
Ingo Gaida ${ }^{1}$, \\ Institut für Physik, Humboldt-Universität, \\ Invalidenstrasse 110, D-10115 Berlin, Germany
}

\begin{abstract}
Global $N=2$ supersymmetry in four dimensions with a gauged central charge is formulated in superspace. To find an irreducible representation of supersymmetry for the gauge connections a set of constraints is given. Then the Bianchi identities are solved subject to this set of constraints. It is shown that the gauge connection of the central charge is a $N=2$ vector multiplet. Moreover the Bogomol'nyi bound of the massive particle states is studied.
\end{abstract}

\footnotetext{
${ }^{1}$ e-mail: gaida@qft2.physik.hu-berlin.de, supported by Cusanuswerk
} 
In this paper, global $N=2$ supersymmetric theories in four dimensions are discussed. Although these theories are not directly related to physics at the electroweak scale, they have been extensively studied in the last years. It started with an analysis of the vacuum structure of $N=2$ supersymmetric gauge theories with gauge group $G=S U(2)$ by Seiberg and Witten, who showed that these theories exhibit physical phenomena like asymptotic freedom, chiral symmetry breaking and confinement of the electric charge [1]. Moreover a version of Olive-Montonen electric-magnetic duality appears [2]. Their results have been extended to other gauge groups [3, [4] and to the case of effective string theories in four dimensions [5]:6].

In the context of the electric-magnetic duality the central charge plays an important role, because the electric and the magnetic charges can appear as a complex central charge in the algebra. Technically these central charges enter a supersymmetric theory because certain surface terms, usually discarded in deriving the algebra, are non-vanishing [7].

The generator of the central charge is defined to commute with all the other generators of the theory [8]. From the point of view of the $N=2$ supersymmetry algebra the appearance of a central charge leads to new torsion terms. As a consequence the Bianchi identities differ from the ones without central charge. The solution of the latter is well known [9].

In this paper the solution of the Bianchi identities with gauged central charge will be given in the context of global $N=2$ supersymmetry. In $N=2$ supergravity the central charge must be gauged anyway [10]. To solve the Bianchi identities one has to find an appropriate set of constraints. These constraints yield an irreducible representation of supersymmetry for the $U(1)$ gauge connections.

It is well known that the central charge can determine the quantum mechanical mass spectrum by the use of the Bogomol'nyi bound [7]. It is shown that the gauging of the central charge can change this mass bound, if the lowest component of the gauge connection of the central charge (Higgs field) has a non-vanishing vacuum expectation value.

The paper is organized as follows: First of all the $N=2$ supersymmetry algebra with gauged central charge is introduced. For simplicity only the case where the central charge is gauged by one $U(1)$ gauge group is considered. Then the set of constraints on the field strengths is given and the solution of the Bianchi identities subject to these constraints is shown. In particular the gauge connection of the central charge is discussed in detail. Moreover the possible Higgs-effect, which is a consequence of the gauging of the central charge, is studied. The whole discussion is given in the framework of rigid $N=2$ superspace.

After the formulation of $N=1$ supersymmetric theories an extended version with two supersymmetry generators has been formulated [11, 12, 13. The corresponding algebra 
ist

$$
\begin{aligned}
\left\{\mathcal{D}_{\alpha}{ }^{i}, \bar{D}_{\dot{\alpha} j}\right\} & =-2 i \delta^{i}{ }_{j} \sigma^{m}{ }_{\alpha \dot{\alpha}} \partial_{m} \\
\left\{\mathcal{D}_{\alpha}{ }^{i}, \mathcal{D}_{\beta}{ }^{j}\right\} & =-2 i g^{i j} \varepsilon_{\alpha \beta} \partial_{z} \\
\left\{\overline{\mathcal{D}}_{\dot{\alpha} i}, \overline{\mathcal{D}}_{\dot{\beta} j}\right\} & =-2 i g_{i j} \varepsilon_{\dot{\alpha} \dot{\beta}} \partial_{\bar{z}}
\end{aligned}
$$

All the other graded commutators vanish. The occurence of the central charge leads to the new torsion $T_{\alpha \beta}^{z i j}=2 g^{i j} \varepsilon_{\alpha \beta}$ in (1.1). Note that the central charge index $z$ is used as an internal index. Gauging this algebra with an abelian gauge group yields

$$
\begin{aligned}
\left\{\mathcal{D}_{\alpha}{ }^{i}, \bar{D}_{\dot{\alpha} j}\right\} & =-2 i \delta^{i}{ }_{j} \sigma^{m}{ }_{\alpha \dot{\alpha}} \mathcal{D}_{m}+i F_{\alpha \dot{\beta}}{ }^{i}{ }_{j} \\
\left\{\mathcal{D}_{\alpha}{ }^{i}, \mathcal{D}_{\beta}{ }^{j}\right\} & =-2 i g^{i j} \varepsilon_{\alpha \beta} \mathcal{D}_{z}+i F_{\alpha \beta}{ }^{i j} \\
\left\{\overline{\mathcal{D}}_{\dot{\alpha} i}, \overline{\mathcal{D}}_{\dot{\beta} j}\right\} & =-2 i g_{i j} \varepsilon_{\dot{\alpha} \dot{\beta}} \mathcal{D}_{\bar{z}}+i F_{\dot{\alpha} \dot{\beta} i j} \\
{\left[\mathcal{D}_{m}, \mathcal{D}_{\alpha}{ }^{j}\right] } & =i F_{m \alpha}{ }^{j(r)} T_{(r)} \\
{\left[\mathcal{D}_{m}, \overline{\mathcal{D}}_{\dot{\alpha} i}\right] } & =i F_{m \dot{\alpha} i}{ }^{(r)} T_{(r)} \\
{\left[\mathcal{D}_{m}, \mathcal{D}_{n}\right] } & =i F_{m n}{ }^{(r)} T_{(r)} \\
{\left[\mathcal{D}_{z}, \mathcal{D}_{A}\right] } & =i F_{z A}{ }^{(r)} T_{(r)} \\
{\left[\mathcal{D}_{\bar{z}}, \mathcal{D}_{A}\right] } & =i F_{\bar{z} A}{ }^{(r)} T_{(r)} .
\end{aligned}
$$

Here the covariant derivatives are given as $\mathcal{D}_{A}=D_{A}+i A_{A}$ with the index $A \sim$ $(m, \alpha i, \dot{\alpha} j, z, \bar{z})$. The generators $T_{(r)}$ of the $U(1)$ gauge group satisfy $\left[T_{(r)}, T_{(s)}\right]=0$ and the superfields $A_{A}=A_{A}^{(r)} T_{(r)}$ represent the $U(1)$ gauge connections. They transform in the adjoint representation under gauge transformations:

$$
A_{A} \rightarrow e^{-i \Lambda} A_{A} e^{i \Lambda}-i e^{-i \Lambda} D_{A} e^{i \Lambda}
$$

It is rather unconventional that the operator $\mathcal{D}_{z}$ of the gauged central charge does not commute with all the generators - in this sense it is not a 'good' central charge.

Now we set the first constraint on the gauge connections: Any gauge connection is independent of the central charge, i.e.

$$
A_{A}=A_{A}\left(x^{m}, \theta_{i}^{\alpha}, \bar{\theta}^{\dot{\alpha} j}\right)
$$

So we have $\Lambda=\Lambda\left(x^{m}, \theta_{i}^{\alpha}, \bar{\theta}^{\dot{\alpha} j}\right)$. and therefore the gauge connection of the central charge transforms now as $A_{z} \rightarrow e^{-i \Lambda} A_{z} e^{i \Lambda}$ under gauge transformations. The same holds for $A_{\bar{z}}$.

\footnotetext{
${ }^{1}$ Here the following conventions for complex conjugation and for the $S U(2)_{R}$ metric $g_{i j}$ is used: $\mathcal{D}_{\alpha}^{i+}=\overline{\mathcal{D}}_{\dot{\alpha} i}, g^{12}=1, g^{i j}=-g^{j i}=-g_{i j}, g^{i j} g_{j k}=\delta^{i}{ }_{k}$. Moreover the conventions of [18] are used.
} 
As usual the field strength is given in general as

$$
F_{A B}=D_{A} A_{B}-(-)^{a b} D_{B} A_{A}+i T_{A B}{ }^{C} A_{C}
$$

and the corresponding Bianchi identities are

$$
\oint_{A, B, C}(-)^{a c}\left\{D_{A} F_{B C}+i T_{A B}^{E} F_{E C}\right\}=0 .
$$

In our specific case with one complex central charge we find 13 field strengths and 26 Bianchi identities after elimination of the trivial ones. To proceed we set constraints on the field strengths to eliminate superfluous component fields. First of all we have the natural constraints:

$$
F_{\alpha \beta}{ }^{i j}=F_{\alpha \dot{\beta}}^{i}{ }_{j}=F_{\dot{\alpha} \dot{\beta} i j}=0
$$

These constraints are natural in the sense that they can be obtained by an appropriate redefinition of the gauge connections appearing in the $N=2$ algebra. Note that these constraints survive the truncation to a $N=1$ supersymmetric theory and lead in that case to the $N=1$ vector multiplet [18]. Second we have the central charge constraints: Some of them follow directly from (1.4).

$$
\begin{aligned}
F_{z \bar{z}}=F_{\dot{\alpha} \bar{z} i}=F_{\alpha z}{ }^{i} & =0 \\
F_{a z}-\partial_{a} A_{z} & =0 \\
F_{a \bar{z}}-\partial_{a} A_{\bar{z}} & =0 \\
F_{\alpha \bar{z}}{ }^{i}-D_{\alpha}{ }^{i} A_{\bar{z}} & =0 \\
F_{\dot{\alpha} z i}-\bar{D}_{\dot{\alpha} i} A_{z} & =0
\end{aligned}
$$

Moreover the number of non-trivial field strengths reduces to 7 and the number of non-trivial constrained Bianchi identities to 21. However, 10 bianchi identities are still fulfilled by the use of the algebra. The other 11 Bianchi identities must be solved subject to the set of constraints. The solution yields the following constraints on the connection of the central charge:

$$
\begin{aligned}
D_{\alpha}^{i} A_{z} & =0 \\
\bar{D}_{\dot{\alpha} i} A_{\bar{z}} & =0 \\
{\left[D^{\alpha i}, D_{\alpha}^{j}\right] A_{\bar{z}} } & =\left[\bar{D}_{\dot{\alpha}}^{i}, \bar{D}^{\dot{\alpha} j}\right] A_{z} \\
{\left[D^{\alpha}{ }_{i}, D_{\alpha j}\right] A_{\bar{z}} } & =\left[\bar{D}_{\dot{\alpha} i}, \bar{D}^{\dot{\alpha}}{ }_{j}\right] A_{z}
\end{aligned}
$$

All the other component fields can be expressed in terms of the gauge connection of the central charge. In this context the following superfield-identities hold: 


$$
\begin{aligned}
F_{\beta \alpha \dot{\alpha}}{ }^{j} & =\varepsilon_{\beta \alpha} \bar{D}_{\dot{\alpha} i} g^{i j} A_{z} \\
F_{\dot{\beta} \alpha \dot{\alpha} j} & =\varepsilon_{\dot{\beta} \dot{\alpha}} D_{\alpha}^{i} g_{i j} A_{\bar{z}} \\
F_{\alpha \dot{\alpha} \beta \dot{\beta}} & =\varepsilon_{\alpha \beta} f_{(\dot{\alpha} \dot{\beta})}+\varepsilon_{\dot{\alpha} \dot{\beta}} f_{(\alpha \beta)}
\end{aligned}
$$

with the definitions

$$
\begin{aligned}
f_{(\alpha \beta)} & =-\frac{i}{4} D_{\alpha}^{i} g_{i j} D_{\beta}^{j} A_{\bar{z}} \\
f_{(\dot{\alpha} \dot{\beta})} & =-\frac{i}{4} \bar{D}_{\dot{\alpha} i} g^{i j} \bar{D}_{\dot{\beta} j} A_{z} .
\end{aligned}
$$

Collecting this information about the gauge connection of the central charge we find that it is a $N=2$ vector multiplet $[9,12,13,14,15]$. Note that another set of constraints might yield a different multiplet.

The $8_{B}+8_{F}$ vector multiplet contains a complex scalar, a vector, a left-handed $S U(2)_{R}$ spinor doublet and a real $S U(2)_{R}$ triplet, which represents three auxiliary fields:

$$
A_{\bar{z}} \sim\left(X_{\bar{z}}, a_{m} \mid \lambda_{\alpha \bar{z}}^{i} \| Y^{i j}\right)
$$

In the following we will refer to the complex scalar as the Higgs field, whereas the spinor doublet represents the Higgsino and the Gaugino. The $S U(2)_{R}$ triplet obeys the constraint:

$$
Y^{i j}=Y^{j i}=\bar{Y}_{i j}
$$

The vector multiplet is defined at component level as

$$
\begin{aligned}
A_{\bar{z} \mid} & =X_{\bar{z}}(x) \\
\frac{i}{8} \varepsilon^{\gamma \beta} \sigma_{m n \beta}{ }^{\alpha} D_{\alpha}{ }^{i} g_{i j} D_{\gamma}{ }^{j} A_{\bar{z}}+\frac{i}{8} \bar{\sigma}_{m n}{ }_{\dot{\alpha}}^{\dot{\beta}} \varepsilon^{\dot{\alpha} \dot{\gamma}} \bar{D}_{\dot{\beta} i} g^{i j} \bar{D}_{\dot{\gamma} j} A_{z \mid} & =f_{m n}(x) \\
D_{\alpha}{ }^{i} A_{\bar{z} \mid} & =\lambda_{\alpha \bar{z}}{ }^{i}(x) \\
{\left[D^{\alpha i}, D_{\alpha}{ }^{j}\right] A_{\bar{z} \mid} } & =Y^{i j}(x) .
\end{aligned}
$$

The field strength of the abelian component vector field is defined in the usual way: $f_{m n}=\partial_{m} a_{n}-\partial_{n} a_{m}$. The component fields transform under supersymmetry transformations generated by the operator $\delta=\xi_{i}^{\alpha} D_{\alpha}^{i}+\bar{\xi}_{\dot{\alpha} i} \bar{D}^{\dot{\alpha} i}$ as follows: 


$$
\begin{aligned}
\delta X_{\bar{z}}= & \xi_{i}^{\alpha} \lambda_{\alpha \bar{z}}{ }^{i} \\
\delta a_{m}= & \frac{1}{2} \bar{\sigma}_{m}{ }^{\alpha}{ }^{\alpha}\left(\xi_{\alpha}{ }^{i} \bar{\lambda}_{\dot{\alpha} z i}+\bar{\xi}_{\dot{\alpha} i} \lambda_{\alpha \bar{z}}{ }^{i}\right) \\
\delta \lambda_{\alpha \bar{z}}{ }^{i}= & \frac{1}{8} \xi_{j}^{\beta} \varepsilon_{\beta \alpha} Y^{j i}-\frac{i}{2} \xi_{j}^{\beta} g^{j i}\left(\sigma^{m n} \varepsilon\right)_{\beta \alpha} f_{m n}-2 i \bar{\xi}_{\dot{\alpha} j} \varepsilon^{\dot{\alpha} \dot{\beta}} g^{j i} \sigma_{\alpha \dot{\beta}}^{m} \partial_{m} X_{\bar{z}} \\
\delta Y^{i j}= & 4 i \xi_{k}^{\alpha}\left(g^{k i} \sigma_{\alpha \dot{\beta}}^{m} \partial_{m} \bar{\lambda}^{\dot{\beta}}{ }_{z}{ }^{j}+g^{k j} \sigma_{\alpha \dot{\beta}}^{m} \partial_{m} \bar{\lambda}^{\dot{\beta}}{ }_{z}{ }^{i}\right) \\
& -4 i \bar{\xi}_{\dot{\alpha} k}\left(g^{k i} \bar{\sigma}^{m \dot{\alpha} \beta} \partial_{m} \lambda_{\beta \bar{z}}{ }^{j}+g^{k j} \bar{\sigma}^{m \dot{\alpha} \beta} \partial_{m} \lambda_{\beta \bar{z}}{ }^{i}\right)
\end{aligned}
$$

The Bogomol'nyi bound of massive particle states is associated with the central charge. Gauging the central charge can change the bound. To show this the inequality for the masses will be derived in a way described in [7]: First Majorana spinors $\mathcal{D}^{i}=$ $\left(\begin{array}{c}\mathcal{D}_{\alpha}^{i} \\ \overline{\mathcal{D}}^{\dot{\alpha} i}\end{array}\right)$ and $\mathcal{D}_{j}^{+}=\left(\overline{\mathcal{D}}_{\dot{\beta} i}, \mathcal{D}^{\beta}{ }_{j}\right)$ are introduced. Then the eigenvalues of the $8 \times 8$ matrix $\left\{\mathcal{D}^{i}, \mathcal{D}_{j}{ }^{+}\right\}$must be calculated by the use of the algebra in the rest frame. With $i \mathcal{D}_{\alpha \dot{\beta}}=-\delta_{\alpha \dot{\beta}} M$ we find that the eigenvalues of the matrix are real if and only if

$$
M^{2} \geq\left|\partial_{z}+i X_{z}\right|^{2}
$$

Of course the eigenvalues of the matrix must be real and that is why the inequality must hold for massive particle states. So a vacuum expectation value of the Higgs field can change the mass bound.

Changing the basis and introducing the $Q_{\alpha}{ }^{i}$ as supersymmetry generators with

$$
\left\{Q_{\alpha}^{i}, \bar{Q}_{\dot{\beta} j}\right\}=2 \sigma_{\alpha \dot{\beta}}{ }^{m} \delta^{i}{ }_{j} P_{m} \quad \text { and } \quad\left\{Q_{\alpha}^{i}, Q_{\beta}^{j}\right\}=2 g^{i j} \varepsilon_{\alpha \beta} Z
$$

we recover the well-known result of Olive and Witten [7]. However, in this basis the effect of gauging the central charge is hidden.

To conclude, it is shown in this paper that the central charge appearing in the $N=2$ algebra in four dimensions can be gauged by abelian gauge groups.

The case for one $U(1)$ gauge group has been studied explicitly by solving the Bianchi identities subject to a set of constraints. It turns out that the gauge connection of the central charge is a $N=2$ vector multiplet with respect to these constraints. For more than one central charge one has additional constraints on the field strengths like $F_{z_{i} z_{j}}=F_{\bar{z}_{i} \bar{z}_{j}}=0$, where the index $i$ labels the number of different central charges. However, all the associated gauge connections of the central charges are $N=2$ vector multiplets.

Then the effect of the gauging concerning the Bogomol'nyi bound of the massive particle states has been investigated. It is shown that a non-vanishing vacuum expectation value of the Higgs field can change this mass bound. As a consequence one can understand from an algebraic point of view, why the breaking of a non-abelian gauge group down 
to $U(1)$ can change the $N=2$ central charge. Just because of the fact that any abelian field strength $F_{\alpha \beta}{ }^{i j}$ appearing in the algebra

$$
\left\{\mathcal{D}_{\alpha}^{i}, \mathcal{D}_{\beta}{ }^{j}\right\}=-2 i g^{i j} \varepsilon_{\alpha \beta} \mathcal{D}_{z}+i F_{\alpha \beta}{ }^{i j}
$$

can enter the gauged central charge. This lead to the natural constraints.

The gauged central charge operator does not commute with all the generators of the theory. This problem cannot be solved in this simple, linearized appoach to supersymmetry. The solution of this problem might be a non-linear realization of $N=2$ supersymmetry or the inclusion of gravity. Anyway, problems with the central charge are well known in $N=2$ supersymmetric gauge theories: The discussion of hypermultiplets, for example, leads off-shell to an infinite set of auxiliary fields.

Finally, it should be mentioned that a $N=2$ supersymmetric theory in four dimensions can be obtained by dimensional reduction of a $N=1$ supersymmetric theory in six dimensions [16,17]. From this point of view the generator of the complex ungauged central charge is related to partial derivatives with respect to the two internal coordinates. Analogous the Higgs field represents two degrees of freedom of the sixdimensional $U(1)$ gauge connection in four dimensions. And the constraint (1.4) makes any four-dimensional gauge connection independent of the internal coordinates. The important difference between the six and the four dimensional theory is that the sixdimensional $U(1)$ gauge connection cannot have a non-vanishing vacuum expectation value. This would violate Lorentz invariance. On the other hand in the four dimensional case the two degrees of freedom of the six-dimensional $U(1)$ gauge connection, which enter the Higgs field, can have a non-vanishing vacuum expectation value.

Acknowledgement: I would like to thank S. Ketov and C. Preitschopf for discussions about the central charge and B. de Wit for helpful correspondence.

\section{References}

1. N. Seiberg and E. Witten, Nucl. Phys. B426 (1994) 19. Electric-magnetic duality, monopole condensation and confinement in $N=2$ supersymmetric Yang-Mills theory

N. Seiberg and E. Witten, Nucl. Phys. B431 (1994) 484. Monopoles, duality and chiral symmetry breaking in $N=2$ supersymmetric $Q C D$

2. C. Montonen and D. Olive, Phys. Lett. 72B (1977) 117. Magnetic monopoles as gauge particles?

3. A. Klemm, W. Lerche, S. Theisen and S. Yankielowicz, Phys. Lett. 344B (1995) 169. Simple singularities and $N=2$ supersymmetric Yang-Mills theory

A. Klemm, W. Lerche and S. Theisen, hep-th (9505150). Non-perturbative effective actions of $N=2$ supersymmetric gauge theories 
4. P. Argyres and A. Faraggi, Phys. Rev. Lett. 74 (1995) 3931. The vacuum structure and spectrum of $N=2$ supersymmetric $S U(N)$ gauge theory

5. B. de Wit, V. Kaplunovsky, J. Louis and D. Lüst, Nucl. Phys. B451 (1995) 53. Perturbative couplings of vector multiplets in $N=2$ heterotic string vacua

G. Lopes Cardoso, D. Lüst and T. Mohaupt, Nucl. Phys. B455 (1995) 131. Non-perturbative monodromies in $N=2$ heterotic string vacua

6. I. Antoniadis, S. Ferrara, E. Gava, K.S. Narain and T.R. Taylor, Nucl. Phys. B447 (1995) 35. Perturbative prepotential and monodromies in $N=2$ heterotic superstring

7. D. Olive and E. Witten, Phys. Lett 78B (1978) 97. Supersymmetry algebras that include topological charges

8. R. Haag, J.T. Lopuszánski and M. Sohnius, Nucl. Phys.B88 (1975) 257. All possible generators of supersymmetries of the S-matrix

9. R. Grimm, M. Sohnius and J. Wess, Nucl. Phys. B133 (1978) 275. Extended supersymmetry and gauge theories

10. C.K. Zachos, Phys. Lett. 76B (1978) 329. $N=2$ supergravity theory with a gauged central charge

B. de Wit, J.W. van Holten and A. Van Proeyen, Phys. Lett. 95B (1980) 51. Central charges and conformal supergravity

P. Claus, B. de Wit, M. Faux, B. Kleijn, R. Siebelink and P. Termonia, hepth 9512143 . The vector-tensor supermultiplet with gauged central charge

11. S. Ferrara and B. Zumino, Nucl. Phys. B79 (1974) 413. Supergauge invariant Yang-Mills theories

12. R.J. Firth and J.D. Jenkins, Nucl. Phys.B85 (1975) 525. Supersymmetry with isospin

13. P. Fayet, Nucl. Phys. B113 (1976) 135. Fermi-Bose Hypersymmetry

14. M. de Roo, B. de Wit, J.W. van Holten and A. Van Proeyen, Nucl. Phys. B173 (1980) 175. Chiral superfields in $N=2$ supergravity

B. de Wit, J.W. van Holten and A. Van Proeyen, Nucl. Phys. B184 (1981) 77, E B222 (1983) 516. Structure of $N=2$ supergravity

E. Cremmer, J.P. Derendinger, C. Kounnas, B. de Wit, S. Ferrara, L. Girardello and A. Van Proeyen, Nucl. Phys. B250 (1985) 385. Vector multiplets coupled to $N=2$ supergravity: Super-Higgs-Effect, flat potentials and geometric structure 
B. de Wit, P.G. Lauwers and A. Van Proeyen, Nucl. Phys. B255 (1985) 596. Lagrangians of $N=2$ supergravity-matter systems

15. M. Müller, Nucl. Phys. B289 (1987) 557. Chiral actions for minimal $N=2$ supergravity

16. L. Brink, J. Schwarz and J. Scherk, Nucl. Phys. B121 (1977) 77. Supersymmetric Yang-Mills theory

17. M.F. Sohnius, K.S. Stelle and P.C. West, Nucl. Phys. B173 (1980) 127. Dimensional reduction by legendré transformation generates off-shell supersymmetric Yang-Mills theory

18. J. Bagger and J. Wess, Supersymmetry and Supergravity, Princeton University Press. 